\title{
Neutron Star Constraints on the H Dibaryont
}

\author{
Norman K. Glendenning and Jürgen Schaffner-Bielich \\ Nuclear Science Division \& Institute for Nuclear and Particle Astrophysics, \\ Lawrence Berkeley National Laboratory, MS: 70A-3307 \\ Berkeley, California 94720
}

July 7, 2018

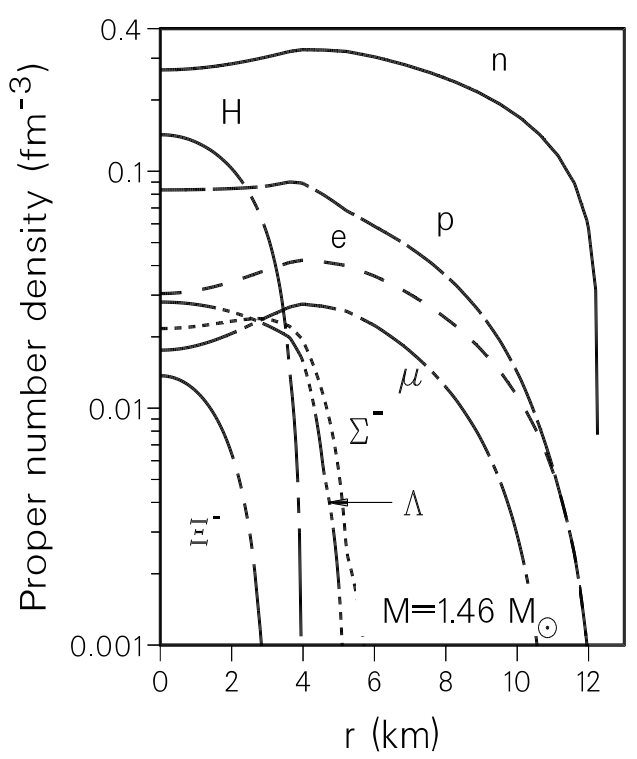

PACS

26.60.+c, 14.20.Pt, 98.38.Mz, 97.60.Jd, 97.60.Gb

\footnotetext{
${ }^{1}$ This work was supported by the Director, Office of Energy Research, Office of High Energy and Nuclear Physics, Division of Nuclear Physics, of the U.S. Department of Energy under Contract DE-AC03-76SF00098.

${ }^{*}$ [nkg.papers]sample.tex, July 7, 2018
} 


\title{
Neutron Star Constraints on the H Dibaryon
}

\author{
Norman K. Glendenning and Jürgen Schaffner-Bielich \\ Nuclear Science Division \& Institute for Nuclear and Particle Astrophysics, \\ Lawrence Berkeley National Laboratory, MS: 70A-3307 \\ Berkeley, California 94720
}

\begin{abstract}
We study the influence of a possible $\mathrm{H}$ dibaryon condensate on the equation of state and the overall properties of neutron stars whose population otherwise contains nucleons and hyperons. In particular, we are interested in the question of whether neutron stars and their masses can be used to say anything about the existence and properties of the $\mathrm{H}$ dibaryon. We find that the equation of state is softened by the appearance of a dibaryon condensate and can result in a mass plateau for neutron stars. If the limiting neutron star mass is about that of the Hulse-Taylor pulsar a condensate of $\mathrm{H}$ dibaryons of vacuum mass $\sim 2.2 \mathrm{GeV}$ and a moderately attractive potential in the medium could not be ruled out. On the other hand, if the medium potential were even moderately repulsive, the $\mathrm{H}$, would not likely exist in neutron stars. If neutron stars of mass $\sim 1.6 M_{\odot}$ were known to exist, attractive medium effects for the $\mathrm{H}$ could be ruled out.
\end{abstract}

\section{Introduction}

Since Jaffe proposed that there may exist a stable dihyperon (a quark composite with baryon number two) [1], an ongoing quest for this particle began [2]. Recent searches using kaon beams [3] or heavy ion beams [4, 5, 6] found no candidates or are still in progress [7]. There exist some claims for evidence for the $\mathrm{H}$ dibaryon produced in proton-nucleus [8] and in heavy-ion collisions [9]. Nevertheless, these candidates might be misidentified $K_{L}^{0}$ as seen in [5]. For a most recent overview on the search for the $\mathrm{H}$ dibaryon we refer to [10].

There are numerous mass estimates for the $\mathrm{H}$ dibaryon and they are reviewed in [11]. The existence or nonexistence of the $\mathrm{H}$ dibaryon is strongly connected with the observation of double $\Lambda$ hypernuclei which has been discussed in [12]. Three double $\Lambda$ hypernuclei have been reported in literature: ${ }_{\Lambda \Lambda}^{6} \mathrm{He}$ [13], ${ }_{\Lambda \Lambda}^{10} \mathrm{Be}$ [14], and ${ }_{\Lambda \Lambda}^{13} \mathrm{~B}$ [15, 16]. The two $\Lambda$ 's can decay by strong interactions to the $H$ dibaryon. As this has not been seen in the above events, the $\mathrm{H}$ must either be heavier than $m_{H}>2 m_{\Lambda}+B_{\Lambda \Lambda} \approx 2.22$ $\mathrm{GeV}$ [17] or the events are misidentified as an $\mathrm{H}$ hypernucleus with a shallow attractive nuclear potential [11]. A more stringent condition is the observation of the weak mesonic decay of the double $\Lambda$ hypernuclei giving $m_{H}>m_{\Lambda}+m_{p}+m_{\pi^{-}}+B_{\Lambda} \approx 2190$ $\mathrm{MeV}$ [18] where $B_{\Lambda}$ depends on the mass of the decay fragment and is $B_{\Lambda}=-3.1$ 
$\mathrm{MeV}$ for ${ }_{\Lambda}^{5} \mathrm{He}$. In all cases, a deeply bound $\mathrm{H}$ dibaryon seems to be ruled out by these events.

If the $\mathrm{H}$ dibaryon exists, it will have a certain impact also on the properties of dense matter. It is quite established nowadays, that neutron stars have a large hyperon fraction in the core and might be described as giant hypernuclei, though bound by gravity [19]. Here again, the presence of hyperons might restrict certain properties of the $\mathrm{H}$ dibaryon. Recently, studies for neutron stars have been done for nuclear matter without hyperons but including $\mathrm{H}$ dibaryon condensation [20] and limits have been set for the coupling constants of the $H$ dibaryon [21].

There might exist heavier partners of the $\mathrm{H}$ dibaryon, lumps of strange quark matter dubbed strangelets. There are several heavy-ion experiments dedicated to search for this novel form of matter [22, 23, 24]. In the MIT bag model, strangelets with $A \leq 6$ are found to be unbound [25]. Nevertheless, light strangelet candidates in the range of $6<A<40$ might be stable against weak hadronic decay [26, 27] (an overview of the properties of strange matter can be found in 28]). The H dibaryon as well as these light strangelets can occur in dense matter as a precursor of the phase transition to a quark plasma.

In this paper, we study the influence of $\mathrm{H}$ dibaryons and other strangelet candidates on the composition and structure of neutron stars including the hyperon degree of freedom. We are particularly interested in the question of whether neutron stars and their masses can be used to say anything about the existence and properties of the $\mathrm{H}$ dibaryon. In section 2, we discuss the condition for the occurrence of dibaryons and strangelets in neutron star matter. The relativistic mean field model with hyperons and the $\mathrm{H}$ dibaryon is presented in section 3 . Implications for a $\mathrm{H}$ dibaryon condensate are discussed in section 1 and summarized in the last section.

\section{Composite Objects in Neutron Star Matter}

Here we discuss the general features of the appearance of composite quark objects in neutron star matter. Nuclei will dissolve in dense matter due to a Mott transition at quite low density. Hence, hypernuclei with similar binding energies will also dissolve. The situation is different for strangelets, if they are energetically favored compared to hadrons. Then strangelets will appear at a certain critical density which will depend on the chemical potentials and the mass of the strangelet. The most stable strangelet candidates will have a closed shell, i.e. they have zero total spin and are bosons. Also the $\mathrm{H}$ dibaryon (consisting of two $\mathrm{u}, \mathrm{d}$, and $\mathrm{s}$ quarks) has zero spin and will form a Bose condensate if it appears in dense matter.

The general condition for a Bose condensation of strangelets is that the effective energy must be equal to the chemical potential:

$$
E_{S}^{*}(k=0)=m_{S}+U(\rho)=B \cdot \mu_{n}-Z \cdot \mu_{e}
$$

where B stands for the baryon number and $\mathrm{Z}$ for the charge of the strangelet with mass $m_{S}$. The corresponding chemical potentials are $\mu_{n}=\mu_{B}$ and $\mu_{e}$ (unit baryon and unit negative electric charge, respectively). $U(\rho)$ is the potential felt by a strangelet 
in a dense environment. Neglecting interaction and modification of the mass in the medium, the threshold condition for the appearance of a strangelet is

$$
\frac{m_{S}}{B}=\mu_{n}-\mu_{e} \frac{Z}{B}
$$

Hence, the baryochemical potential determines the onset of condensation as the charge to baryon ratio is between +2 and -1 and the electrochemical potential is much weaker.

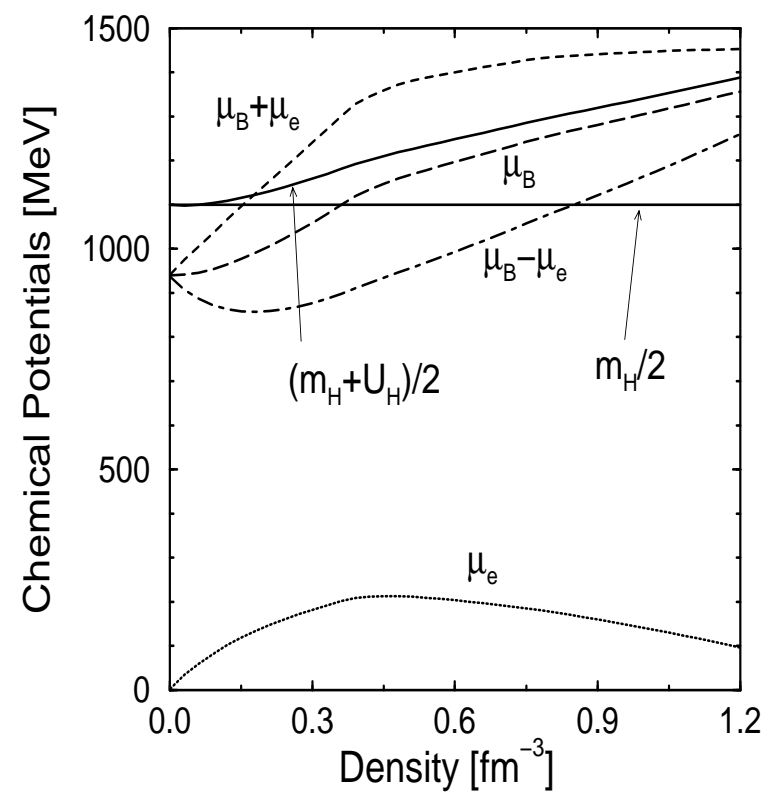

Figure 1: The chemical potentials per baryon number for a strangelet with a charge of $Z / A=-1\left(\mu_{B}+\mu_{e}\right), 0\left(\mu_{B}\right)$, and +1 $\left(\mu_{B}-\mu_{e}\right)$. The horizontal line is the $\mathrm{H}$ dibaryon mass of $m_{H}=$ $2200 \mathrm{MeV}$ assumed to be density independent. The crossing of this line with the line for $\mu_{B}$ at $\rho_{c} \approx 0.36 \mathrm{fm}^{-3}$ marks the onset of $\mathrm{H}$ dibaryon condensation. The $\mathrm{H}$ mass with a medium dependent repulsive potential of $U_{H}=30 \mathrm{MeV}$ at $\rho_{0}$ is also shown by the curve $\left(m_{H}+U_{H}\right) / 2$.

We illustrate threshold conditions for the $\mathrm{H}$ dibaryon (or any other strangelet with assumed free mass per baryon of $1.1 \mathrm{GeV}$ ) in Fig. 1 under two circumstances: (1) free dibaryon, and (2) dibaryon whose mass is modified by the medium. The two solid curves represent the dibaryon mass per baryon under these two circumstances. More generally, the chemical potentials for a strangelet with $Z / B=-1,0$, and +1 are also plotted. The point where these lines intersect and rise above the mass per nucleon for a particular strangelet marks the density threshold above which the strangelet in 
question would comprise one of the constituents of matter. The threshold for free and medium modified dibaryons (or strangelets) of free mass per baryon of $1.1 \mathrm{GeV}$ can be read from the intersections. Nucleons, leptons as well as hyperons are included in the composition of matter. The effect of hyperons is clearly evident in Fig. 1 through their saturating effect on $\mu_{e}$ at $\rho \approx 0.4 \mathrm{fm}^{-3}$. The other chemical potentials accordingly increase less rapidly with density above the hyperon thresholds.

The lowest mass strangelet is the $\mathrm{H}$ dibaryon which is bound due to color magnetic forces [1]. Assuming the $\mathrm{H}$ has a mass of $m_{H}=2.2 \mathrm{GeV}$ and does not change in the medium, gives a critical density of about $2 \rho_{0}$. Note that a negatively charged candidate appears at a much lower density and for the assumed mass, would be a constituent of matter at densities above $\rho \approx 0.16 \mathrm{fm}^{-3}$. If the $\mathrm{H}$ dibaryon feels a repulsive potential at saturation density of $U_{H}\left(\rho_{0}\right)=30 \mathrm{MeV}$ then the critical density is shifted beyond the maximum density reached in the interior of a neutron star for that equation of state. Note that for a slightly smaller repulsive potential or for matter without hyperons the $\mathrm{H}$ dibaryon will appear (because its chemical potential $\mu_{B}$ will rise above its mass as modified by the medium).

A similar analysis can be done for other strangelet candidates. However, strangelets with mass numbers of $B \leq 6$ are not stable due to a repulsive color magnetic interaction except for the $\mathrm{H}$ dibaryon [25]. Nevertheless, strange dibaryon states have been predicted to be bound in a relativistic quark potential model [29]. Negatively charged candidates are e.g. $\Sigma^{-} \Sigma^{-}$and $\Xi^{-} \Xi^{-}$which are heavier than the $\mathrm{H}$ dibaryon but might appear at a similar density as the $\mathrm{H}$ due to their negative charge. It has been also proposed in the MIT bag model that negatively charged strangelets with closed shells are likely to be most stable against strong and weak emission of hadrons [27]. The candidates with a closed shell found are e.g. for $B=10, Z=-4$, for $B=12, Z=-6$, and $B=16, Z=-10$. The masses are not precisely known as they depend crucially on the value of the bag parameter. To be metastable, their masses per baryon should lie between $m_{\Xi}$ and $m_{n}$. (Absolute stability is unlikely because of the finite size shell effect on the quark wave functions.) Mass estimates ranges between $m_{S} / B=1.04-1.24 \mathrm{GeV}$ for the above candidates. ¿From Fig. 1, the negatively charged strangelets would appear in neutron stars at densities above $\approx 0.4 \mathrm{fm}^{-3}$ if their masses are lower than

$$
\frac{m_{S}}{B} \leq \mu_{B}^{\max }+\frac{1}{2} \mu_{e}^{\max } \approx 1.23 \mathrm{GeV}
$$

(assuming no interactions). Therefore, if strangelets do not feel too high a repulsive potential in the medium they can appear as a Bose condensate in neutron stars. In the following we will discuss the modification of the properties of neutron stars due to the appearance of a strangelet condensate. We choose to study the case of the $\mathrm{H}$ dibaryon as it is the lightest candidate and might appear first in dense matter.

\section{Mean-Field Model with Dibaryons}

First note that the $\mathrm{H}$ dibaryon is a boson with zero spin and isospin. We use the standard extended $\sigma-\omega-\rho$ model to describe the baryon sector interacting through 
the mesons

$$
\begin{aligned}
\mathcal{L}= & \sum_{B} \bar{\Psi}_{B}\left(i \gamma_{\mu} \partial^{\mu}-m_{B}+g_{\sigma B} \sigma-g_{\omega B} \gamma_{\mu} V_{\mu}-g_{\rho B} \vec{\tau}_{B} \vec{R}_{\mu}\right) \Psi_{B}+\frac{1}{2} \partial_{\mu} \sigma \partial^{\mu} \sigma-\frac{1}{2} m_{\sigma}^{2} \sigma^{2} \\
& -U(\sigma)-\frac{1}{4} V_{\mu \nu} V^{\mu \nu}+\frac{1}{2} m_{\omega}^{2} V_{\mu} V^{\mu}+U(V)-\frac{1}{4} \vec{R}_{\mu \nu} \vec{R}^{\mu \nu}+\frac{1}{2} m_{\rho}^{2} \vec{R}_{\mu} \vec{R}^{\mu}
\end{aligned}
$$

where $B$ is summed over all states of the baryon octet, the scalar meson is denoted by $\sigma$, the vector mesons are denoted by $V_{\mu}$ and $R_{\mu}$ for the iso-scalar and iso-vector meson and $V_{\mu \nu}=\partial_{\mu} V_{\nu}-\partial_{\nu} V_{\mu}$. We have taken into account possible self-interaction terms for the scalar field $U(\sigma)$ [30] and for the vector field [31]

$$
U(\sigma)=\frac{1}{3} b m\left(g_{\sigma} \sigma\right)^{3}+\frac{1}{4} c\left(g_{\sigma} \sigma\right)^{4}, \quad U(V)=\frac{d}{4}\left(V_{\mu} V^{\mu}\right)^{2} .
$$

The $\mathrm{H}$ dibaryon is coupled to the mean fields by a minimal coupling scheme following 20]:

$$
\mathcal{L}_{D}=\mathcal{D}_{\mu}^{*} H^{*} \mathcal{D}^{\mu} H-m_{H}^{*} H^{*} H
$$

where the vector fields are coupled via the standard replacement

$$
\mathcal{D}_{\mu}=\partial_{\mu}+i g_{\omega H} V_{\mu}
$$

This ensures consistency with Ward identities, i.e. the vector fields are coupled to a conserved current. The effective mass of the $\mathrm{H}$ is defined as in the baryon case

$$
m_{H}^{*}=m_{H}-g_{\sigma H} \sigma
$$

This gives, as for the vector field, a quadratic coupling term of the $\mathrm{H}$ to the scalar field in the Lagrangian (6). It turns out, that with this choice of coupling, the scalar and vector density for the $\mathrm{H}$ are the same in the mean field approximation. It was also shown that this model is thermodynamically consistent [20]. The equation of motion is simply

$$
\left[\mathcal{D}_{\mu}^{*} \mathcal{D}^{\mu}+m_{H}^{* 2}\right] H(\omega, \vec{k})=0
$$

For s-wave condensation $(\vec{k}=0)$ one gets the dispersion relation

$$
\omega_{H}=m_{H}^{*}+g_{\omega H} V_{0}=\mu_{H}=2 \mu_{B}
$$

in the mean field approximation, where $\mu_{B}$ is the baryochemical potential. This relation fixes the amplitude of the $\mathrm{H}$ dibaryon condensate. The density of the $\mathrm{H}$ dibaryon is increased until the effective energy of the $\mathrm{H}$ dibaryon is equal to its chemical potential. Note that this implies that there must be a repulsive potential between the $\mathrm{H}$ dibaryons at a certain $\mathrm{H}$ dibaryon density. Otherwise, the effective energy is decreasing with increasing $\mathrm{H}$ dibaryon density and it will never attain its chemical potential.

We do not repeat the full set of equations for the baryons as they can be found in e.g. [19] in detail. We note the additional terms due to the $\mathrm{H}$ dibaryon condensate in the equations of motion

$$
m_{\sigma}^{2} \sigma+\frac{\partial}{\partial \sigma} U(\sigma)=\sum_{B} g_{\sigma B} \rho_{s}^{B}+2 g_{\sigma H} m_{H}^{*} H^{*} H
$$




$$
m_{\omega}^{2} V_{0}+d V_{0}^{3}=\sum_{B} g_{\omega B} \rho_{V}^{B}+2 g_{\omega H}\left(\mu_{H}-g_{\omega H} V_{0}\right) H^{*} H
$$

Here one needs to define only one density for the $\mathrm{H}$ dibaryon due to the dispersion relation (10)

$$
\rho_{H}=2 m_{H}^{*} H^{*} H=2\left(\mu_{H}-g_{\omega H} V_{0}\right) H^{*} H .
$$

The $\mathrm{H}$ dibaryon contributes to the energy density in the form

$$
\epsilon_{H}=2 m_{H}^{*} H^{*} H=m_{H}^{*} \rho_{H}
$$

but contributes to the pressure only indirectly through the modification of the meson fields via the additional terms in the equations of motion.

\subsection{Baryon-Meson interactions}

There exists various parameterizations in the literature for the nucleon-nucleon interactions in the mean field model. The parameters are either fixed by nuclear matter properties or fitted to properties of spherical nuclei. For example, the parameter set used in 32 with scalar self-interactions $U(\sigma)$ corresponds to the nuclear matter properties: $B / A=16.3 \mathrm{MeV}, \rho_{0}=1.53 \mathrm{fm}^{-3}, a_{\text {sym }}=32.5 \mathrm{MeV}, K=240 \mathrm{MeV}$, $m^{*} / m=0.78$ (which for brevity we refer to as GM91). The parameter set TM1 33] has been fitted to the binding energy, radii, and surface thickness of heavy nuclei. The latter model has a self-interaction term for the vector field $U(V)$. This set has been used in [34. We adopt these two models as guidelines in the following.

The hyperon coupling constants have also been chosen differently. We will consider two cases: (1) universal coupling of the hyperons and (2) coupling constants using $\mathrm{SU}(6)$ relations. In the former case, all hyperons are coupled equally [32

$$
\frac{g_{\sigma Y}}{g_{\sigma}}=\frac{g_{\rho \Sigma}}{g_{\rho}}=\frac{g_{\rho \Xi}}{g_{\rho}}=0.6
$$

where $Y$ stands for the hyperons $\Lambda, \Sigma$, and $\Xi$. Note that the $\Lambda$ has isospin zero, the $\Sigma$ has isospin 1 , while the $\Xi$ and nucleon have isospin $1 / 2$. This gives an additional factor of 2 for the $\rho-\Sigma$ term in the Lagrangian and a vanishing iso-vector coupling constant for the $\Lambda$.

In the other case, $\mathrm{SU}(6)$ relations [35] are used for the vector coupling constants of the hyperons

$$
g_{\omega}: g_{\omega \Lambda}: g_{\omega \Sigma}: g_{\omega \Xi}=3: 2: 2: 1
$$

which scale according to the number of light quarks of the baryon. The iso-vector coupling constants scale with the isospin like in the universal case but are fixed differently to the nucleon iso-vector coupling constant. In our notation this means

$$
g_{\rho}=g_{\rho \Sigma}=g_{\rho \Xi}, \quad g_{\rho \Lambda}=0
$$

The SU(6) symmetry takes already care of the isospin so that the notation as used in 34

$$
g_{\rho}: g_{\rho \Lambda}: g_{\rho \Sigma}: g_{\rho \Xi}=1: 0: 2: 1
$$


means the same. It reflects the strength of the iso-vector potentials of the baryons but starts then from a different, $\mathrm{SU}(3)$-symmetric Lagrangian.

Both cases, universal and $\mathrm{SU}(6)$, are consistent with $\Lambda$ hypernuclear data insofar as the potential depth of the $\Lambda$ in normal nuclear matter is fixed to its phenomenological value of $U_{\Lambda}\left(\rho_{0}\right)=-30 \mathrm{MeV}$. For the universal case the vector coupling constants are adjusted to this potential depth for all hyperons. For the SU(6) case the scalar coupling constants are adjusted. Note that in addition, the SU(6) coupling scheme (quark model) is successful in describing the small $\Lambda$-hypernuclear spin-orbit splitting [36].

\subsection{Dibaryon interactions}

The value of the coupling constants of the $\mathrm{H}$ to the scalar and the vector field, $g_{\sigma H}$ and $g_{\omega H}$ are unknown. They must satisfy two constraints: (I) the H should not appear in normal nuclear matter, and (II) the interaction should allow for neutron star masses at least as large as the well established mass of the Hulse-Taylor pulsar.

The simple quark counting rule suggests that $g_{\omega H} / g_{\omega N}=4 / 3$ as the $\mathrm{H}$ has four light quarks. This choice is motivated from the success of using $\mathrm{SU}(6)$ (quark model)

relations for hyperons in describing hypernuclear properties [35, 36]. We use it to fix $g_{\omega H}$. Having fixed the vector coupling without regard to the above constraints (I) and (II), the burden of satisfying the constraints falls on the scalar coupling (against the background of the other couplings defining the Lagrangian of the theory).

To fix the scalar coupling $g_{\sigma H}$ we consider the following. A range for the scalar coupling constant $g_{\sigma H}$ can be determined from values of the $\mathrm{H}$ potential in the medium at $\rho_{0}$

$$
U_{H}=-g_{\sigma H} \sigma+g_{\omega H} V_{0}
$$

because the meson fields are known. The $\mathrm{H}$ potential at $\rho_{0}$ should not be deeper than

$$
U_{H}\left(\rho_{0}\right)>2 E_{F}-m_{H}=2\left(m_{N}-16 \mathrm{MeV}\right)-m_{H} \approx-350 \mathrm{MeV}
$$

else the $\mathrm{H}$ dibaryon would condense at normal nuclear matter density. We choose specific discrete values in the range of $U_{H}\left(\rho_{0}\right)=+30,0,-30 \mathrm{MeV}$. To each, a specific value of $g_{\sigma H}$ is implied through Eq. (18). We will find that potentials deeper than $-30 \mathrm{MeV}$ would decrease the limiting neutron star mass below observed masses (at least in the parameterizations of the Lagrangian that we have considered).

The energy of the $\mathrm{H}$ dibaryon is plotted in Fig. 2 for neutron star matter including hyperons for the various $\mathrm{H}$ potentials. The $\mathrm{H}$ dibaryon feels a repulsive potential above normal nuclear density irrespective of the chosen potential at $\rho_{0}$. The repulsive high density behavior arises from the interaction of the $\mathrm{H}$ and the vector meson. This repulsion generally dominates at high density unless the scalar interaction is too strong. We discuss this issue below. The slope at high density is quite similar for the set GM91 and the curves for the different $\mathrm{H}$ potentials are just shifted. The vector potential dominates at high density and is chosen to be the same $(4 / 3$ that of the nucleon) in all three cases giving the same slope at high density. The shift comes from the differences in the scalar potential which saturates at high density. 


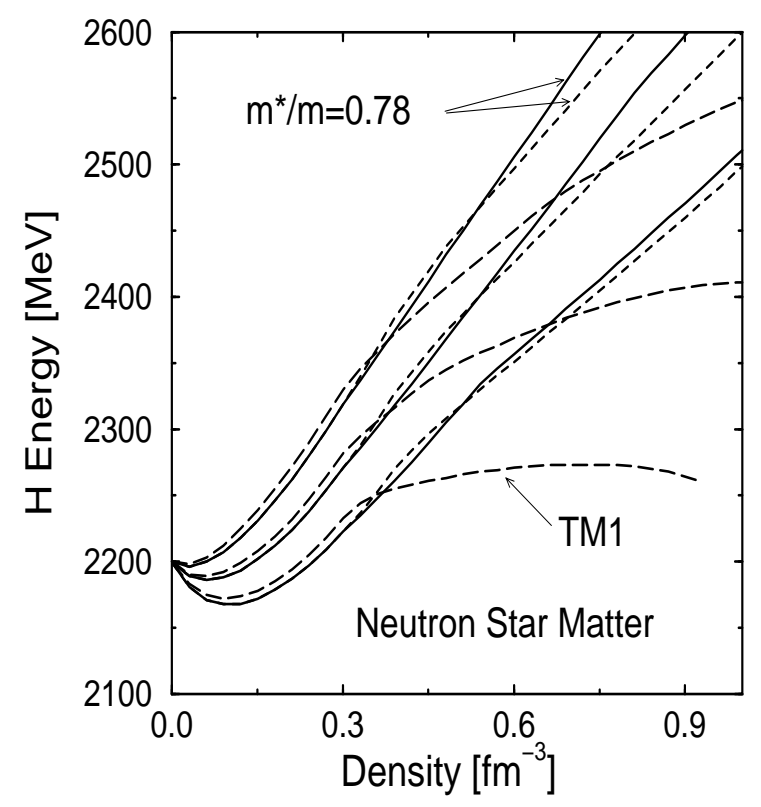

Figure 2: The energy of the $\mathrm{H}$ dibaryon in neutron star matter as a function of baryon density with $U_{H}\left(\rho_{0}\right)=-30,0,+30 \mathrm{MeV}$ from bottom to top. Solid lines stand for the parameter set GM91 using universal couplings, dotted for $\mathrm{SU}(6)$ couplings, dashed lines for the set TM1.

The behavior at high density is quite different for the parameter set TM1. Here the vector potential has a nonlinear dependence on the baryon density due to the vector field selfinteraction terms. Note that the $\mathrm{H}$ energy and thus the baryon chemical potential stays rather constant with density for the lowest curve $\left(U_{H}\left(\rho_{0}\right)=-30\right.$ $\mathrm{MeV}$ ). It is especially clear from Fig. 2 that the vacuum mass of the $\mathrm{H}$ is not as crucial to the appearance of the $\mathrm{H}$ in neutron stars as its interactions with vector and scalar mesons.

In place of the above considerations for fixing a range for the scalar coupling constant, Faessler et al. 20] invoked the condition

$$
\frac{g_{\sigma H}^{2}}{m_{\sigma}^{2}}<\frac{g_{\omega H}^{2}}{m_{\omega}^{2}}
$$

on the grounds that otherwise the Yukawa potential between $\mathrm{H}$ dibaryons would yield a negative compressibility. This is true at low density. For example, for the parameter set GM91 we find that $\mathrm{H}$ matter is unstable against compression at low density when $U_{H}\left(\rho_{0}\right)<+2 \mathrm{MeV}$. For the parameter set TM1 the low density instability arises when $U_{H}\left(\rho_{0}\right)<-10 \mathrm{MeV}$. However, the situation is more complicated. Even if the equation of state has a negative slope at low density, it can become positive at higher density. Whether or not, cannot be stated in terms of the inequality of Eq. (20) but 
involves all of the other interactions and particle types in matter. Test of the low density behavior is therefore insufficient.

Because gravity compresses a star, the question of stability arises for condensed matter, not for low density matter. For the above quoted models the scalar and vector potentials have a different (nonlinear) behavior at high density which can alter the low-density conclusion. This is demonstrated in Figure 3. The binding energy of pure $\mathrm{H}$ dibaryon matter is shown for the two parameter sets and the three different choices of the $\mathrm{H}$ dibaryon potential in nuclear matter. One sees that the compressibility, which is proportional to the slope of the curves, is negative at low density for $U_{H}\left(\rho_{0}\right)=-30$ $\mathrm{MeV}$ for both parameter sets. Nevertheless, the scalar self-interactions provide a nonlinear dependence of the scalar potential on the density (here $\mathrm{H}$ dibaryon number density). This results in an overall repulsive potential for pure $\mathrm{H}$ matter at higher density and a minimum around normal nuclear density. Hence $\mathrm{H}$ matter can be stable at high density even if the low density limit seems to indicate an instability.

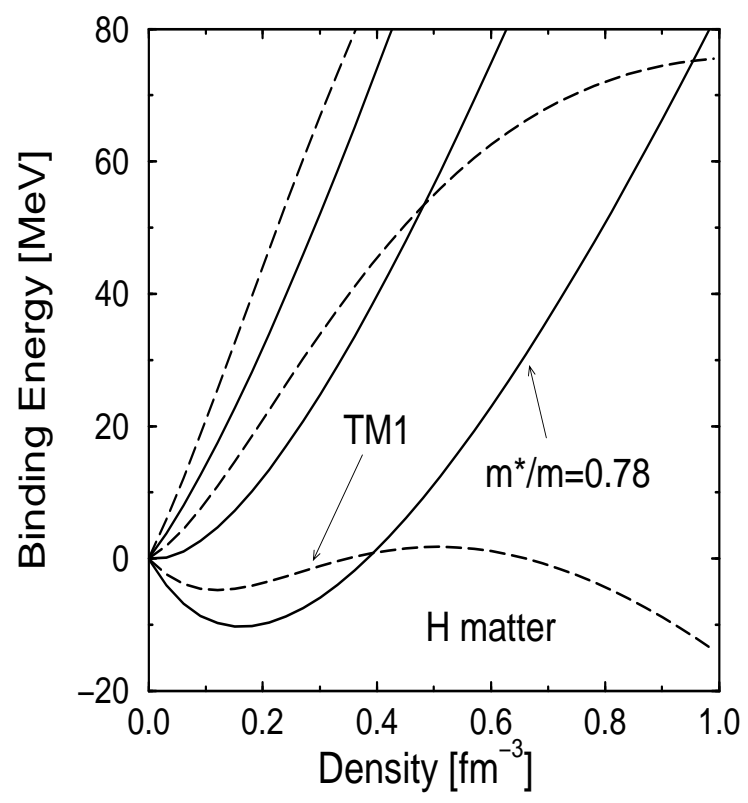

Figure 3: The equation of state of pure $\mathrm{H}$ matter for various potential depths in nuclear matter $\left(U_{H}\left(\rho_{0}\right)=-30,0,+30 \mathrm{MeV}\right.$ from bottom to top). Solid lines stand for the parameter set GM91, dashed lines for TM1.

With reference to Fig. 3, we see that even more complicated situations can arise. For the parameter set TM1, the vector self-interactions cause the energy density due to the vector potential to rise like $\rho^{1 / 3}$ instead of like $\rho$, as for the linear behavior in the standard Walecka model. It can then happen that the scalar potential wins ultimately over the vector potential at very high density. Such a case is seen in 
Figure 3 for the lowest dashed curve: the equation of state has a local minimum around normal nuclear density but the compressibility becomes negative again at higher density.

The instability for pure $\mathrm{H}$ matter does not mean that a neutron star with a $\mathrm{H}$ condensate is unstable against compressional modes, as the overall compressibility can still be positive. This will depend on the intrinsic stiffness of the equation of state used and the hadron population inside the neutron star. A recent analysis considered neutron star matter consisting of nucleons and leptons only [21] and found rather stringent conditions for the coupling constant of the $\mathrm{H}$ dibaryon. The appearance of hyperons already at $(2-3) \rho_{0}$ [19] will certainly alter their conclusions and will be discussed in the following.

\section{H Dibaryons in Neutron Stars}

\subsection{Populations}

To give an early impression of the possible presence of $\mathrm{H}$ dibaryons in neutron stars under acceptable conditions as to (1) its absence in normal matter, and (2) an acceptable value for the limiting mass neutron star, we compare the populations of the

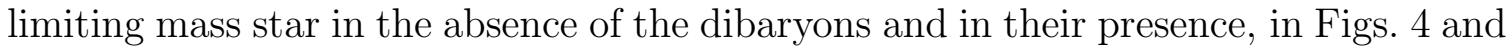
5. Hyperons appear abundantly in the interior of the neutron star. The hyperons $\Lambda$ and $\Xi^{-}$reach values close to the proton density in the stellar core. Protons are more abundant once the negatively charged hyperons $\Sigma^{-}$and $\Xi^{-}$are present to compensate

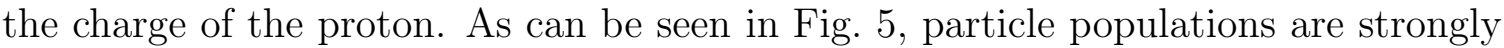
modified in the core of the star where the dibaryon appears. The proton population is suppressed since baryon number is carried more in the $\mathrm{H}$ bosons. Likewise the hyperon populations are strongly suppressed. This is not to say that these baryons have little influence on the $\mathrm{H}$ presence. It is in matter containing significant $\Sigma^{-}$and $\Lambda$ populations that the $\mathrm{H}$ threshold is attained. Outside the core, beyond $r \approx 4 \mathrm{~km}$, populations and the stellar radius are hardly changed by the presence of the $\mathrm{H}$ in the core and therefore cooling of the star would be little effected by the $\mathrm{H}$. $\mathrm{H}$ dibaryons could lie within the star while providing no direct sign of their presence.

\subsection{Limits on the $\mathrm{H}$ dibaryon mass}

Generally the maximum mass of a neutron star is lowered due to $\mathrm{H}$ dibaryon condensation because the condensate does not contribute directly to the pressure and it removes the pressure due to two baryons per dibaryon in the condensate. The equation of state is thereby softened. As the maximum mass should be not lower than $1.44 M_{\odot}$ (the mass of the Hulse-Taylor pulsar), one can try to impose constraints on the medium mass of the $\mathrm{H}$ dibaryon. For the nuclear parameterization TM1, we show the equation of state corresponding to several values of $U_{H}\left(\rho_{0}\right)$ in Fig. 6. The equation of state can be considerably softened by the condensation of $\mathrm{H}$ dibaryons. Especially for the case $U_{H}\left(\rho_{0}\right)=-30 \mathrm{MeV}$ the pressure stays nearly constant once 


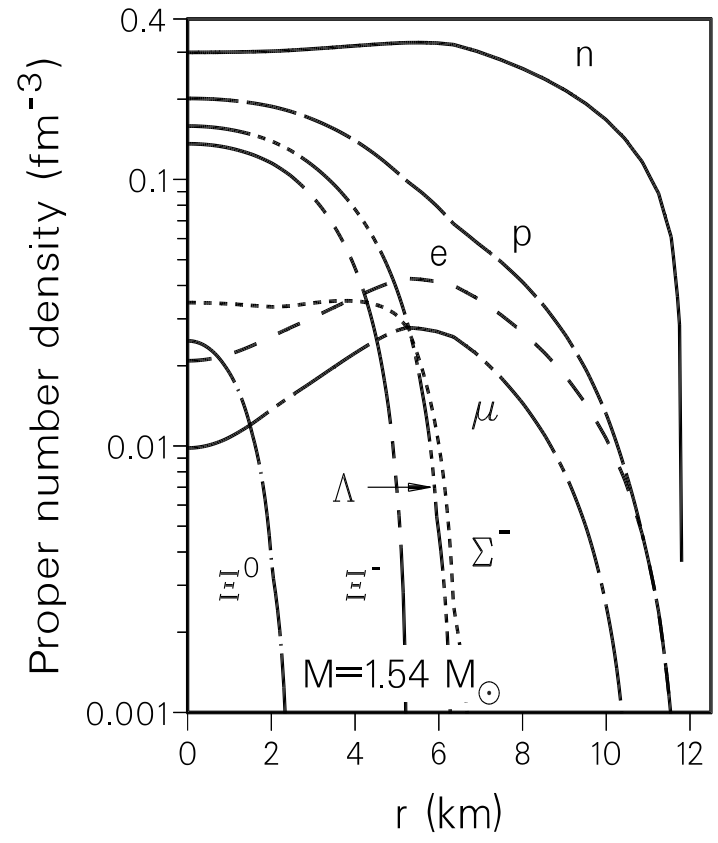

Figure 4: Populations of octet baryons and leptons in a limiting mass star with nuclear properties as described by the case GM91 with hyperon couplings chosen as in the $\mathrm{SU}(6)$ scheme.

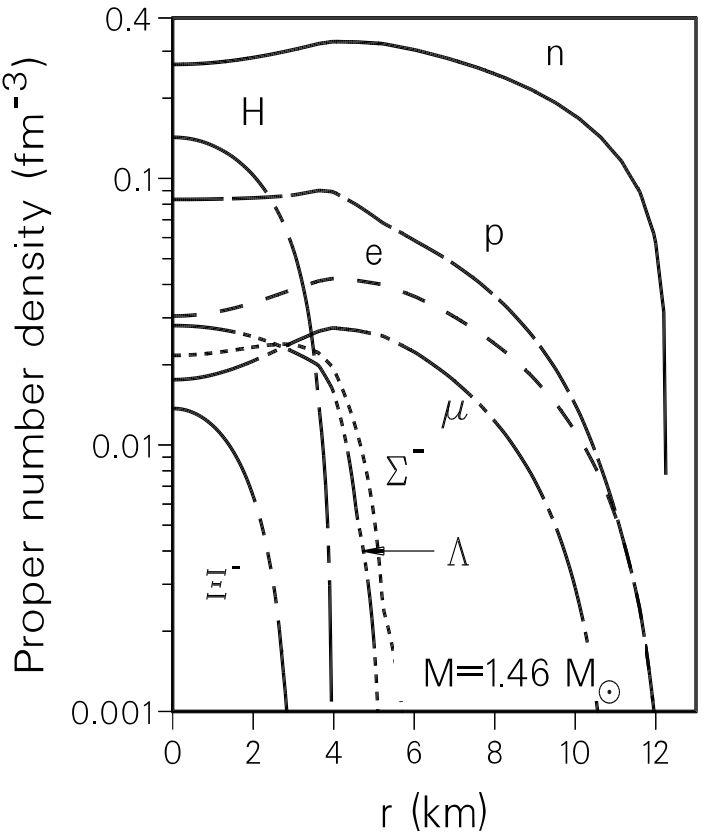

Figure 5: Similar to Fig. 甘 but with the $\mathrm{H}$ dibaryon experiencing a potential of $U_{H}\left(\rho_{0}\right)=-30 \mathrm{MeV}$.

condensation starts. The $\mathrm{H}$ boson constitutes a large fraction of the matter but does not contribute to the pressure directly. The plateau seen in the equation of state in Fig. 6 can be traced back to the equation of state for pure $\mathrm{H}$ dibaryon matter in Fig. 3 (see the bottom dashed curve).

Figure 7 summarizes the neutron star masses for the two hyperon coupling schemes $\mathrm{SU}(6)$ and 'universal' (see section 3.1) and for various values of the interaction $U_{H}\left(\rho_{0}\right)$. The more attractive the interaction, the more populous the dibaryon, the softer the equation of state and the smaller the limiting mass. The potential $U_{H}\left(\rho_{0}\right)=-30$ $\mathrm{MeV}$ is about as attractive as is compatible with the Hulse-Taylor pulsar. For the $\mathrm{SU}(6)$ coupling, $\mathrm{H}$ dibaryons feeling $U_{H}\left(\rho_{0}\right)=0 \mathrm{MeV}$ would not be present in the stable members of the sequence, while for universal coupling of hyperons, the dibaryon is present in small number and reduces the limiting mass marginally by $\approx 0.03 M_{\odot}$. A repulsive dibaryon interaction in the medium would therefore assure its minimal presence if not its total absence (as is the case for the $\mathrm{SU}(6)$ coupling).

One might infer from the figure, that very attractive potentials for the $\mathrm{H}$ of $U_{H}\left(\rho_{0}\right)<-30 \mathrm{MeV}$ can be ruled out by neutron star data. Nevertheless, such a conclusion must be moderated by our ignorance of the $\mathrm{H}$ dibaryon mass. The appearance of the $\mathrm{H}$ condensation depends on the in-medium potential at high density 


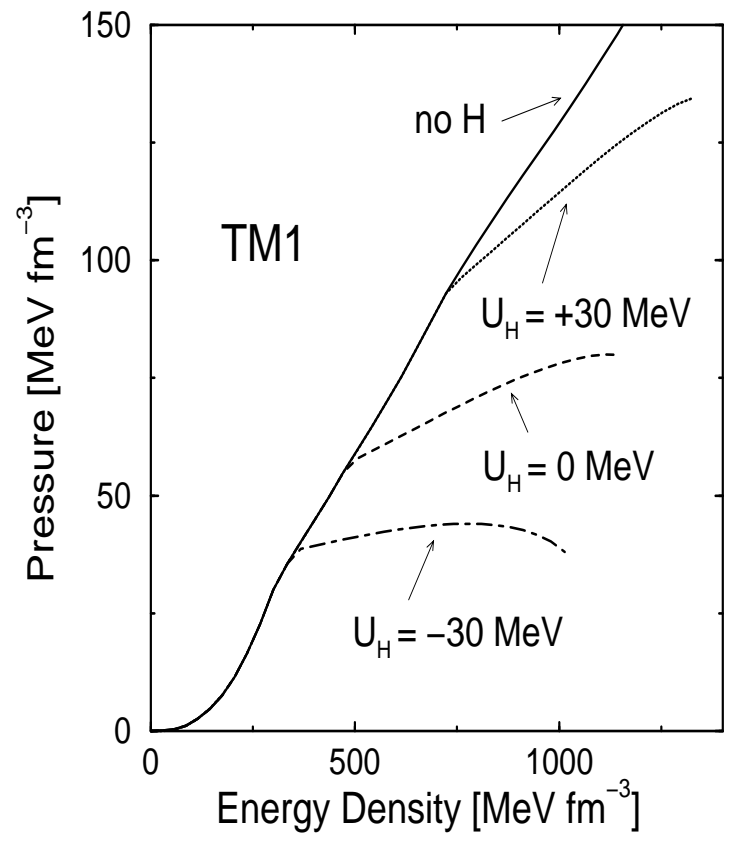

Figure 6: Equation of state for the parameter set TM1, SU(6) coupling for hyperons and several values of the $\mathrm{H}$ dibaryon potential $U_{H}\left(\rho_{0}\right)$.

and the mass of the $\mathrm{H}$ dibaryon which are unknown.

In addition to uncertainties in the vacuum and medium mass of the $H$, there are uncertainties in the underlying nuclear equation of state especially at densities above normal nuclear density. This can be seen by contrasting Fig. 7 with Fig. 8. In the second case the nuclear parameterization is TM1 of section 3.1. Here we see that the $\mathrm{H}$ is present provided $U_{H}\left(\rho_{0}\right)<+30 \mathrm{MeV}$. For an attractive potential of $U_{H}\left(\rho_{0}\right)=-30$ $\mathrm{MeV}$, one finds that the mass of the neutron star reaches a plateau, i.e. the mass of the neutron star is independent of the central energy density. The maximum mass is then $1.44 M_{\odot}$. This is the lowest value allowed by present observation. The plateau comes from the fact, that the equation of state has a nearly constant pressure for the particular coupling due to the appearance of the $\mathrm{H}$ dibaryon. A more attractive potential than $U_{H}\left(\rho_{0}\right)=-30 \mathrm{MeV}$ would result in a negative curvature of the pressure and therefore in an unstable equation of state. Note, that this behavior is mainly related to the potential between the $\mathrm{H}$ dibaryons as discussed in connection with pure $\mathrm{H}$ dibaryon matter (see Figure 3). Neutron star matter is stabilized against collapse to $\mathrm{H}$ dibaryon condensate because of the repulsive interaction with the vector meson provided the $\mathrm{H}$ dibaryon density is not too large. The presence of the other baryons in the matter tends to stabilize the system compared to pure $\mathrm{H}$ matter.

In the present approach one can now exclude certain regions of the assumed mass of the $\mathrm{H}$ Dibaryon $m_{H}$ and its potential at saturation density $U_{H}$. For a too low $\mathrm{H}$ 


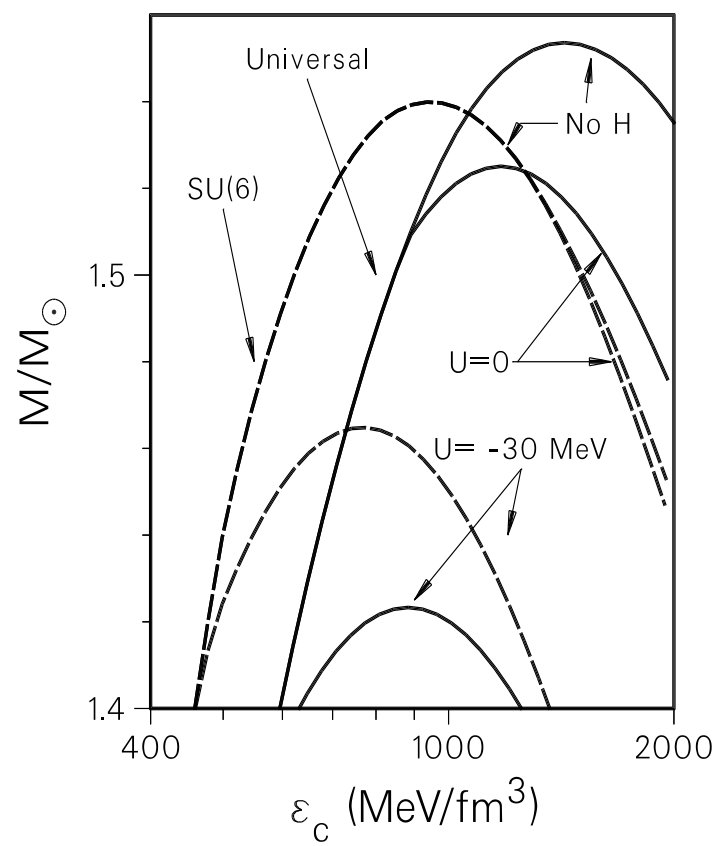

Figure 7: Details of neutron star sequences near the limiting mass for the nuclear parameterization GM91 and two hyperon coupling schemes labeled 'universal' and $\mathrm{SU}(6)$ and for each of these, several values of the dibaryon interaction $U_{H}\left(\rho_{0}\right)$.

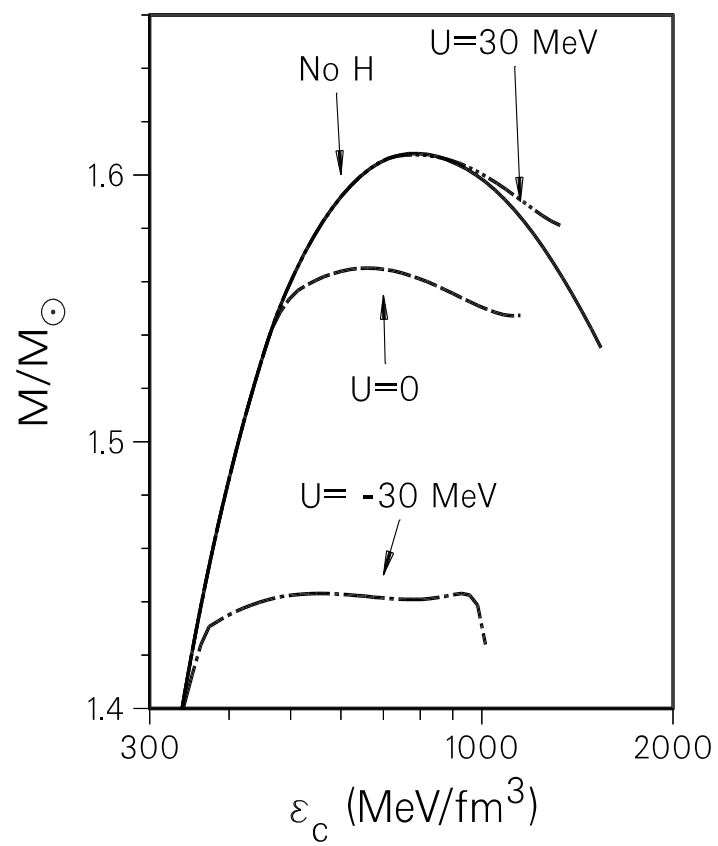

Figure 8: Similar to Fig. 7 but for the nuclear coupling denoted by TM1.

mass or a too deep potential the presence of the $\mathrm{H}$ dibaryon condensate will lower the maximum mass of a neutron star below the observed limit of $1.44 M_{\odot}$. This excluded region is shown as a shaded area in Fig. 9 in an $\left(U_{H}, m_{H}\right)$ diagram for the parameter set GM91 using universal coupling. The dashed line denotes the case for the parameter set TM1. The thresholds for $\Lambda \Lambda$ and $\Sigma \mathrm{N}$ decay which are relevant for the lifetime of the $\mathrm{H}$ dibaryon [37] are also indicated. The results for the two parameterizations are quite close to each other despite their different high density behavior. For an $\mathrm{H}$ mass of lower than $2.13 \mathrm{GeV}$ (the $\Sigma \mathrm{N}$ threshold) an attractive potential in nuclear matter gives too low maximum neutron star mass. Hence, rather long-lived $\mathrm{H}$ dibaryons $\left(\tau>10^{-7} \mathrm{~s}\right.$ according to [37]) are unlikely to form bound $\mathrm{H}$ hypernuclear states. On the other hand, attractive potentials lower than $U_{H}\left(\rho_{0}\right)<-50 \mathrm{MeV}$ seem to be ruled out by neutron star mass constraints for the $\mathrm{H}$ mass range considered. Otherwise, the $\mathrm{H}$ dibaryon has to be heavier than the $2 \Lambda$ threshold and will be a resonance state. These limits will depend also on the chosen vector coupling constant. We are using an effective model and the extrapolation to high density might be completely different in reality. 


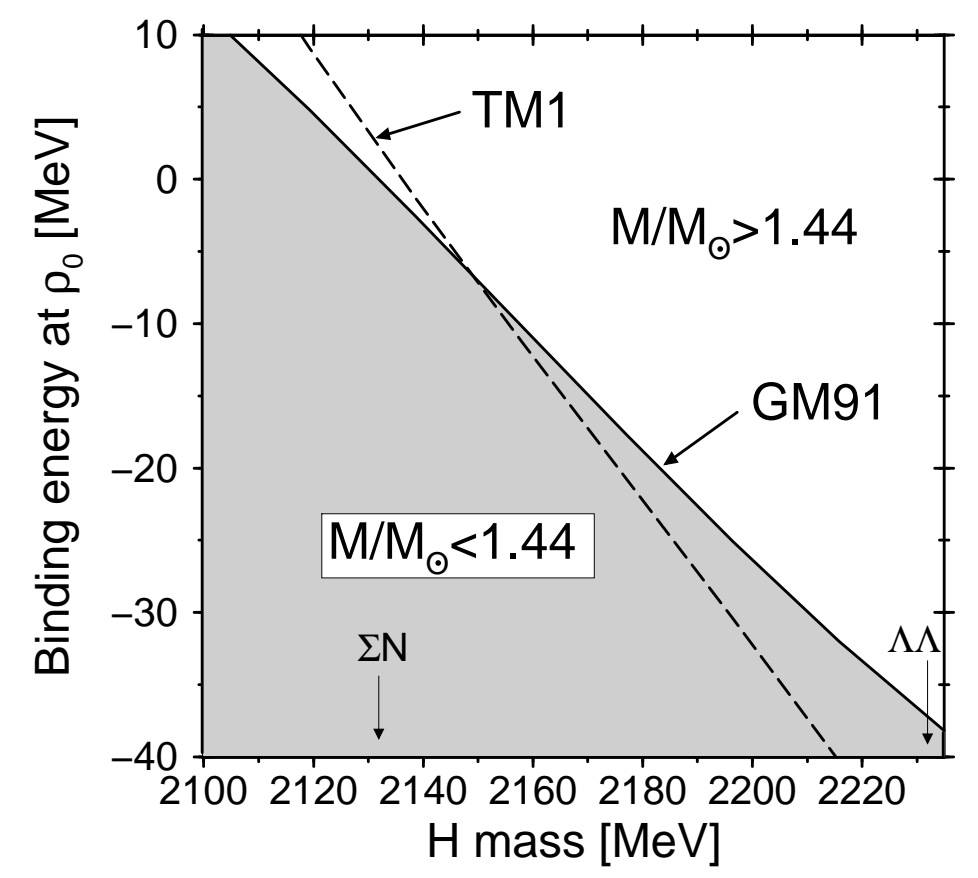

Figure 9: Diagram of the $\mathrm{H}$ vacuum mass and the $\mathrm{H}$ binding energy in normal nuclear matter. The shaded region gives a maximum neutron star mass lower than $1.44 M_{\odot}$ for the parameter set GM91 using universal coupling, the dotted line denotes the case for TM1.

\subsection{Radius and the Mass-Radius Relation}

The mass-radius relation for the GM91 model with universal hyperon couplings is shown in Fig. 10 for several values of the dibaryon potential $U_{H}\left(\rho_{0}\right)$. The more attractive the potential, the softer the equation of state and the lower the limiting mass, as remarked earlier. The radius of the limiting star decreases the more attractive the potential $U_{H}\left(\rho_{0}\right)$ because the star, having less mass, is less gravitationally compacted. Similar results are shown in Fig. 11 for the nuclear parameterization TM1. In this case the limiting mass stars have substantially larger radii.

It is interesting to note, that the presence of the $\mathrm{H}$ dibaryon in neutron stars seems to lower the maximum mass but increases the minimum radius. The mass-radius relation just stops at the point where the $\mathrm{H}$ dibaryon condensation sets in. This is contrary to kaon condensation [38, 39] where the radius decreases for a kaon condensed star. Note that kaon condensation produces a strong phase transition of first order but the equation of state has no plateau if treated in a thermodynamically consistent way [40]. In addition, baryons are not replaced by a baryon number carrying condensate but neutrons are replaced by protons and $K^{-}$'s resulting in a different mass-radius relation. 


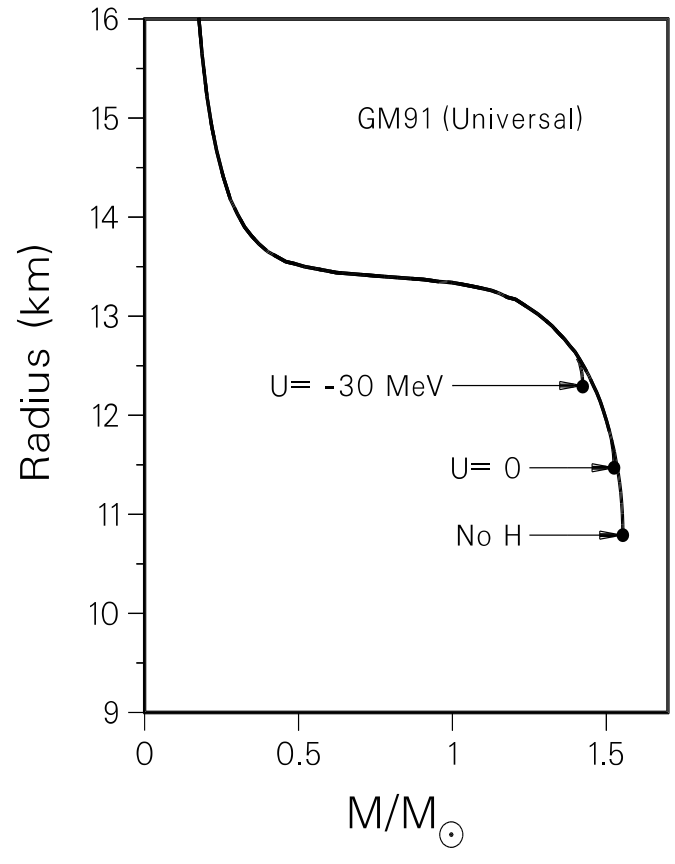

Figure 10: Mass-radius relation for the nuclear model GM91, universal hyperon coupling and several values of the dibaryon potential $U_{H}\left(\rho_{0}\right)$.

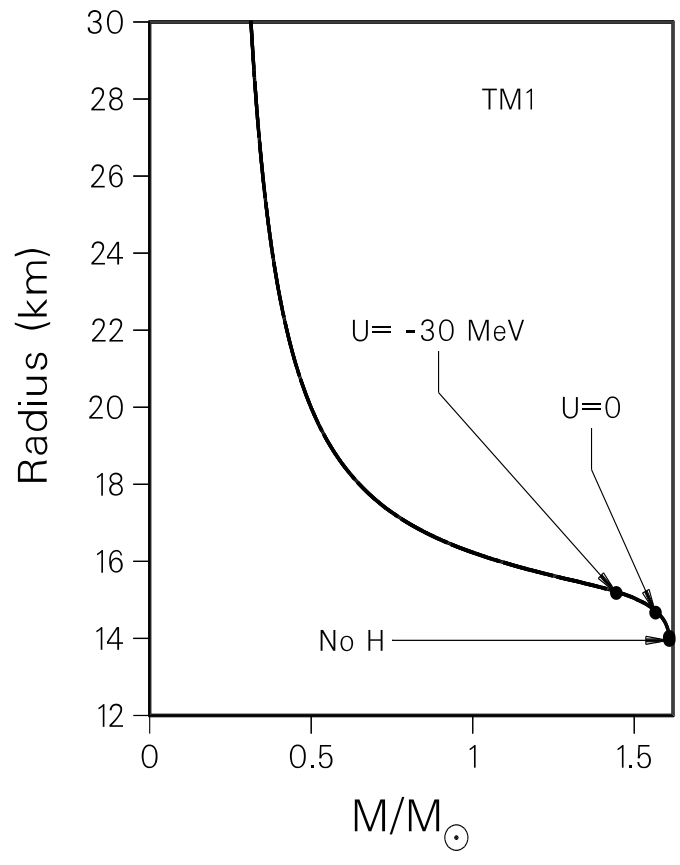

Figure 11: Similar to Fig. 10 but for the nuclear model TM1.

\section{Summary}

We are particularly interested in the question of whether neutron stars and their masses can be used to say anything about the existence and properties of the $\mathrm{H}$ dibaryon. We have studied the influence of the possible occurrence of an $\mathrm{H}$ dibaryon condensate and strangelets in neutron stars including hyperons. Without in-medium modifications, it is quite likely that especially negatively charged strangelets, if they exists, will be present in the dense interior of neutron stars.

The appearance of $\mathrm{H}$ dibaryons in the stellar core depends crucially on their mass and on the chosen potential of the $\mathrm{H}$ in nuclear matter. Hyperons tend to shift the onset of the $\mathrm{H}$ to higher density or to prevent $\mathrm{H}$ dibaryon condensation. If the condensation happens and if the potential of the $\mathrm{H}$ is attractive enough to provide a substantial number density in the neutron star, the maximum mass of the neutron star is reduced compared to the case without the $\mathrm{H}$ dibaryon. The decrease of the maximum mass is moderate and allows for the presence of $\mathrm{H}$ dibaryons in the interior of neutron stars in accord with present neutron star mass data. If the $\mathrm{H}$ dibaryon feels an attractive potential in matter, it can lead to a plateau in the mass of neutron stars, as there exist a region of very slowly rising pressure with energy density.

If the limiting neutron star mass is about that of the Hulse-Taylor pulsar a condensate of $\mathrm{H}$ dibaryons of vacuum mass $\sim 2.2 \mathrm{GeV}$ and a moderately attractive potential 
in the medium could not be ruled out. On the other hand, if the medium potential were even moderately repulsive, the $\mathrm{H}$, would not likely exist in neutron stars. If neutron stars of mass $\sim 1.6 M_{\odot}$ were known to exist, attractive medium effects could be ruled out. For a mass limit of $1.44 M_{\odot}$, attractive potentials for an $\mathrm{H}$ mass below the $\Sigma \mathrm{N}$ threshold $(1.3 \mathrm{GeV})$ are ruled out.

$\mathrm{H}$ dibaryon or strangelet condensation might happen as a precursor to the phase transition to a quark plasma. In this respect, we note that this phase transition is of first order 41]. Hence, small bubbles of strange quark matter will appear in the mixed phase which are most likely negatively charged due to the isospin potential of the nuclear matter. This is in line with the results presented here. As the most stable strangelets have spin zero, the onset to a quark plasma will be initiated by a Bose condensation of strangelets (possibly including the $\mathrm{H}$ dibaryon). As the phase transition proceeds, the bubbles will overlap and will finally replace nuclear matter by essentially filling up the whole volume.

Acknowledgments:

J.S.B. acknowledges support by the Alexander-von-Humboldt Stiftung with a Feodor-Lynen fellowship. This work is supported by the Director, Office of Energy

Research, Office of High Energy and Nuclear Physics, Nuclear Physics Division of the U.S. Department of Energy under Contract No. DE-AC03-76SF00098.

\section{References}

[1] R. L. Jaffe, Phys. Rev. Lett. 38, 195, 617(E) (1977).

[2] S. A. Carroll et al., Phys. Rev. Lett. 41, 777, 1002(E) (1978).

[3] S. Aoki et al., Phys. Rev. Lett. 65, 1729 (1990).

[4] J. Belz et al., Phys. Rev. Lett. 76, 3277 (1996).

[5] J. Belz et al., Phys. Rev. C 56, 1164 (1997).

[6] R. W. Stotzer et al., Phys. Rev. Lett. 78, 3646 (1997).

[7] H. J. Crawford (E896 collaboration), Nucl. Phys. A in press (1998).

[8] B. A. Shahbazian, T. A. Volokhovskaya, V. N. Emelyanenko, and A. S. Martynov, Phys. Lett. B 316, 593 (1993).

[9] R. S. Longacre et al., Nucl. Phys. A 590, 477c (1995).

[10] Proceedings of the International Conference on Hypernuclear and Strange Particle Physics (HYP'97), Nucl. Phys. A to be published (1998).

[11] C. B. Dover, Nuovo Cim. 102A, 521 (1989). 
[12] R. H. Dalitz et al., Proc. Roy. Soc. Lond. A426, 1 (1989).

[13] M. Danysz et al., Nucl. Phys. 49, 121 (1963).

[14] D. J. Prowse, Phys. Rev. Lett. 17, 782 (1966).

[15] S. Aoki et al., Prog. Theor. Phys. 85, 1287 (1991).

[16] C. B. Dover, D. J. Millener, A. Gal, and D. H. Davis, Phys. Rev. C 44, 1905 (1991).

[17] B. O. Kerbikov, Sov. J. Nucl. Phys. 39, 516 (1984).

[18] R. L. Jaffe, Nucl. Phys. B (Proc. Suppl.) 24B, 8 (1991).

[19] N. K. Glendenning, Astrophys. J. 293, 470 (1985).

[20] A. Faessler, A. J. Buchmann, and M. I. Krivoruchenko, Phys. Lett. B 391, 255 (1997).

[21] A. Faessler, A. J. Buchmann, and M. I. Krivoruchenko, Phys. Rev. C 56, 1576 (1997).

[22] T. A. Armstrong et al., Phys. Rev. Lett. 79, 3612 (1997).

[23] D. Beavies et al., Phys. Rev. Lett. 75, 3078 (1995).

[24] G. Applequist et al., Phys. Rev. Lett. 76, 3907 (1996).

[25] A. T. M. Aerts, P. J. G. Mulders, and J. J. de Swart, Phys. Rev. D 17, 260 (1978).

[26] E. P. Gilson and R. L. Jaffe, Phys. Rev. Lett. 71, 332 (1993).

[27] J. Schaffner-Bielich, A. Diener, C. Greiner, and H. Stöcker, Phys. Rev. C 55, 3038 (1997).

[28] C. Greiner and J. Schaffner, Int. J. Mod. Phys. E 5, 239 (1996).

[29] T. Goldman et al., Systematic Theoretical Search for Dibaryons in a Relativistic Model, nucl-th/9803002, 1998.

[30] J. Boguta and A. R. Bodmer, Nucl. Phys. A 292, 413 (1977).

[31] A. R. Bodmer, Nucl. Phys. A 526, 703 (1991).

[32] N. K. Glendenning and S. A. Moszkowski, Phys. Rev. Lett. 67, 2414 (1991).

[33] Y. Sugahara and H. Toki, Nucl. Phys. A 579, 557 (1994).

[34] J. Schaffner and I. N. Mishustin, Phys. Rev. C 53, 1416 (1996). 
[35] C. B. Dover and A. Gal, Progr. Part. Nucl. Phys. 12, 171 (1984).

[36] M. Chiapparini, A. O. Gattone, and B. K. Jennings, Nucl. Phys. A 529, 589 (1991).

[37] J. F. Donoghue, E. Golowich, and B. R. Holstein, Phys. Rev. D 34, 3434 (1986).

[38] V. Thorsson, M. Prakash, and J. M. Lattimer, Nucl. Phys. A 572, 693 (1994).

[39] H. Fujii, T. Maruyama, T. Muto, and T. Tatsumi, Nucl. Phys. A 597, 645 (1996).

[40] J. Schaffner-Bielich and N. K. Glendenning, Strange Phases in Neutron Stars, Proceedings of the Workshop on Nuclear Astrophysics, Hirschegg, Austria, 1998, nucl-th/9802030.

[41] N. K. Glendenning, Phys. Rev. D 46, 1274 (1992). 